\title{
Gonadotropin-releasing hormone agonists during chemotherapy for ovarian function and fertility preservation for patients with early-stage breast
} cancer

This article was published in the following Dove Press journal:

Cancer Management and Research

\author{
Hikmat Abdel-Razeq \\ Department of Internal Medicine, Section \\ of Hematology and Medical Oncology, \\ King Hussein Cancer Center and School \\ of Medicine, University of Jordan, \\ Amman, Jordan
}

\begin{abstract}
Breast cancer remains the most common cancer among women worldwide. Many patients, especially in our region, are affected while young and during their child-bearing years. Chemotherapy, more commonly used in this age group, may result in premature ovarian failure and thus negatively impact their fertility. Several fertility-preservation methods are currently in use in this age group. Unfertilized ova cryopreservation and in vitro fertilization plus embryo cryopreservation are widely used in clinical practice. More recently, ovarian tissue cryopreservation is gaining in popularity. Several clinical trials and metaanalyses have shown that premenopausal women who received ovarian function suppression with gonadotropin-releasing hormone agonists while on chemotherapy were less likely to experience ovarian failure and had higher rates of menses resumption compared to those who did not. Some studies have also shown higher rates of successful pregnancies among treated patients. Given the conflicting results of the reported clinical trials and even the many published meta-analyses, this approach continues to be controversial and should only be used when other established fertility preservation methods cannot be utilized. The current review seeks to provide an updated summary on this controversial topic by reviewing all recently published clinical trials and meta-analyses.
\end{abstract}

Keywords: fertility preservation, breast cancer, pregnancy, premenopausal patients, gonadotropin-releasing hormone agonists, premature ovarian failure

\section{Introduction}

Breast cancer continues to be the most common cancer encountered among young women worldwide. ${ }^{1,2}$ Many patients, especially in our region, are affected while young and during their child-bearing years. ${ }^{3}$ Compared to older patients, breast cancer in younger age groups is usually associated with poor pathological features that reflect negatively on the overall prognosis. ${ }^{4,5}$ Given these clinical and pathological features, chemotherapy remains a cornerstone incorporated in the treatment plans of most such patients.

Chemotherapy regimens used for breast cancer are usually associated with longterm sequelae and impaired quality of life. ${ }^{6}$ In addition, it may result in premature ovarian failure (POF) and infertility, especially among those older than 35 years treated with alkylating agents. ${ }^{7,8}$ In one study, the use of either CMF (cyclophosphamide, methotrexate and 5-fluorouracil) or CEF (cyclophosphamide, epirubicin
Correspondence: Hikmat Abdel-Razeq Department of Internal Medicine, Section of Hematology and Medical Oncology, King Hussein Cancer Center, School of Medicine, University of Jordan, 202 Queen Rania Al Abdullah St., P.O. Box: 1269, Amman I194I, Jordan Tel +962 530 0460. Ext: 1000 Email habdelrazeq@khcc.jo 
and 5-fluorouracil), alone or with endocrine therapy in young women with breast cancer, increased the risk of premature menopause from $<5 \%$ to $>40 \%$. ${ }^{9}$

In another study from the Memorial Sloan-Kettering Cancer Center, researchers looked at the rates of chemotherapy-induced amenorrhea among 235 premenopausal women with early-stage breast cancer. All were 40 years or younger treated with adjuvant anthracycline and taxane-based chemotherapy. Amenorrhea was reported in 15\% of 166 evaluable patients who received chemotherapy and in $17 \%$ of those who received both chemotherapy and tamoxifen. Older women had a significantly higher rate of amenorrhea. ${ }^{10}$

Several methods are in use in routine clinical practice to enhance fertility in this age group. Unfertilized ova cryopreservation and in vitro fertilization plus embryo cryopreservation are widely available. More recently, ovarian tissue cryopreservation is becoming a popular approach but is not a standard yet. ${ }^{11}$ However, its cost might be a barrier, especially in developing countries where insurance and cancer treatment funds may not cover this service.

Given the nature of cancer as an illness, patients are usually overwhelmed by, and focused exclusively on, the cancer diagnosis and active treatment. Patients and families may be concerned that pursuing fertility preservation might delay their treatment, thus negatively affecting their cure rates. Additionally, patients may not be aware of their potential fertility loss, and such a problem may not be communicated well by the treating physicians. ${ }^{12}$

In 2018, the American Society of Clinical Oncology (ASCO) issued an updated guideline recommending that providers discuss fertility preservation with all patients of reproductive age who will be receiving treatment with a possible risk of iatrogenic infertility. ${ }^{13}$ However, compliance with such recommendations continues to be a problem. In one retrospective study, among 303 women with breast cancer aged 40 years or younger who were treated with chemotherapy and/or endocrine therapy, only $80(26 \%)$ had a documented fertility discussion with their physician; $71(89 \%)$ of them sought further fertility preservation options. $^{14}$

In a survey study of young female cancer survivors, lack of knowledge about options for fertility preservation, feeling too distressed or overwhelmed at the time of diagnosis, and concerns about cost were some of the barriers to undergoing fertility preservation. ${ }^{15}$

Several clinical trials and meta-analyses have shown that premenopausal women who received ovarian function suppression with gonadotropin-releasing hormone (GnRH) agonists while on chemotherapy were less likely to have ovarian failure and had higher rates of menses resumption compared to patients who did not. However, many other studies failed to confirm these findings.

\section{Methods}

The PubMed/Medline site was searched for all published literature using the following key words: fertility preservation, breast cancer, pregnancy, premenopausal patients and gonadotropin-releasing hormone agonists. The clinical trials website (www.clinicaltrials.gov) was also searched for all registered clinical trials utilizing the same key words. Clinical trials that included patients with diseases other than breast cancer will not be presented.

\section{Gonadotropin-releasing hormone agonists}

Gonadotropin-releasing hormone agonists are medications used to lower both gonadotropins and sex hormone levels. They are commonly used to lower sex hormone levels in the treatment of hormone-sensitive cancers like breast and prostate cancers. They are widely used as fertility medicine and for certain gynecological disorders such as heavy menses and endometriosis.

With their higher receptor affinity and reduced susceptibility to enzymatic degradation, GnRH agonists are a lot more potent than the natural GnRH molecule. ${ }^{16}$ They bind to the GnRH receptors on pituitary gonadotropinproducing cells, causing a transient release of both luteinizing hormone (LH) and follicle stimulating hormone (FSH). It usually takes 1 week of therapy for the GnRH receptors to be downregulated along with a decline in the pituitary production of both $\mathrm{LH}$ and $\mathrm{FSH}^{17}$

Several formulations of $\mathrm{GnRH}$ agonists are approved for parenteral administration and available on the market, including leuprolide, goserelin, triptorelin, buserelin, and histrelin.

\section{Mechanism of protection}

The rationale behind using ovarian suppression to protect ovarian function during chemotherapy is not clear. Ovarian toxicity and potential failure are expected to be higher if chemotherapy is given while the ovaries are active. In a very old study, Rivkees et al analyzed 30 studies that evaluated gonadal function after combination chemotherapy for Hodgkin's disease and acute lymphocytic leukemia 
or cyclophosphamide therapy for renal diseases. Chemotherapy-induced damage was more likely to occur in patients who were treated when sexually mature compared with those who were treated when prepubertal. These data suggest that chemotherapy-induced damage is proportional to gonadal activity. ${ }^{18}$

In an animal study, Ataya et al prospectively showed that $\mathrm{GnRH}$ agonists protected the ovary against cyclophosphamide-induced damage in rhesus monkeys by significantly decreasing the number of follicles lost during the chemotherapy. During the treatment period, $64.6 \pm 2.8 \%$ of the total primordial follicles were lost in the cyclophosphamide group compared to only $28.9 \pm 9.1 \%$ in the GnRH agonists + cyclophosphamide group $(P<0.05) .{ }^{19}$

Other researchers have hypothesized that the hypogonadotropic state, created by GnRH agonists, decreases the number of primordial follicles entering the differentiation stage, which are more vulnerable to chemotherapy. ${ }^{20}$

Other researchers have suggested that GnRH agonist administration will prevent the expected increase in FSH concentration induced by the negative feedback of low estrogen and inhibin levels and thus may rescue the follicles from accelerated atresia. ${ }^{21}$

In another animal model, Kitajima et al showed that high estrogen concentrations significantly increased ovarian perfusion and vessel endothelial area, and this effect was significantly inhibited, in a dose-dependent way, by the administration of a GnRH agonist (leuprolide). ${ }^{22}$ Thus, the hypoestrogenic state decreases ovarian perfusion, and subsequent reduced delivery of chemotherapy to the ovaries might be considered another possible hypothesis.

\section{Surrogate endpoints}

Several efficacy endpoints and different time frames have been utilized in clinical trials. Six months, 1 and 2 years or even 5-year amenorrhea rates are commonly used endpoints. Postmenopausal levels of FSH and serum estradiol (E2) were occasionally used as additional markers for POF in such patients. Given the lack of close correlations between menses resumption and pregnancy, many of the published clinical trials also looked at pregnancy rates following chemotherapy as a secondary endpoint.

Because of the potential concerns about the outcomes related to hormonal manipulation resulting from the use of such agents, especially while treating a type of tumor that is often hormone-sensitive, researchers also looked at safety endpoints including both disease-free survival (DFS) and overall survival (OS), and some of these clinical trials limited enrollment to women with hormone receptor-negative breast cancer as detailed below.

\section{Published clinical trials}

A number of randomized clinical trials evaluating the use of GnRH agonists to minimize ovarian toxicity induced by chemotherapy have been published, and some were recently updated (Table 1). The possibility of publication bias has to be considered when addressing this issue, too. Most of the trials included patients regardless of their hormone-receptor status, while a few restricted patients' enrollment to those with hormone-receptor negative tumors to avoid any possible impact on cancer treatment outcomes due to hormone manipulations.

The ZIPP (Zoladex In Premenopausal Patients) was a relatively old but large study that included a total of 260 evaluable premenopausal breast cancer patients with a median age of 45 years. Patients were randomly assigned to receive 2 years of goserelin, goserelin plus tamoxifen, tamoxifen alone or no endocrine treatment. Goserelin was given simultaneously with chemotherapy and continued for a total of two years. The chemotherapy regimen was cyclophosphamide, methotrexate and 5-fluorouracil (CMF). Endocrine treatment was given with chemotherapy regardless of the tumor's hormone receptor status. One year after the completion of both CMF and endocrine therapy, goserelin was associated with significantly higher menses recovery (36\%) compared to the control groups $(10 \%), P=0.006$. However, this effect was not observed in the combined tamoxifen and goserelin group. ${ }^{23}$ It is important to highlight here that the study design does not reflect the current clinical practice; $\mathrm{CMF}$ is not commonly used, a median age of 45 is too high to consider fertility preservation, and the pattern and dose of tamoxifen are not in use anymore.

In another study from Egypt, 78 evaluable patients with early-stage breast cancer were randomized to receive adjuvant chemotherapy (anthracyclines and cyclophosphamide) alone or combined with a GnRH agonist. Goserelin was started 2 weeks before chemotherapy and then continued every 4 weeks for a total of 6 months. In the GnRH agonisttreated group, $89.6 \%$ resumed menses compared to only $33.3 \%$ in the chemotherapy-alone group $(P<0.001)$. The authors also reported rates of spontaneous ovulation, $69.2 \%$ vs $25.6 \%(P<0.001)$, in favor of the $\mathrm{GnRH}$ agonist group. Serum estradiol (E2) was significantly higher in the study group $(279 \pm 23.32 \mathrm{pg} / \mathrm{mL}$ versus $75.43 \pm 18.98 \mathrm{pg} / \mathrm{mL}$, 


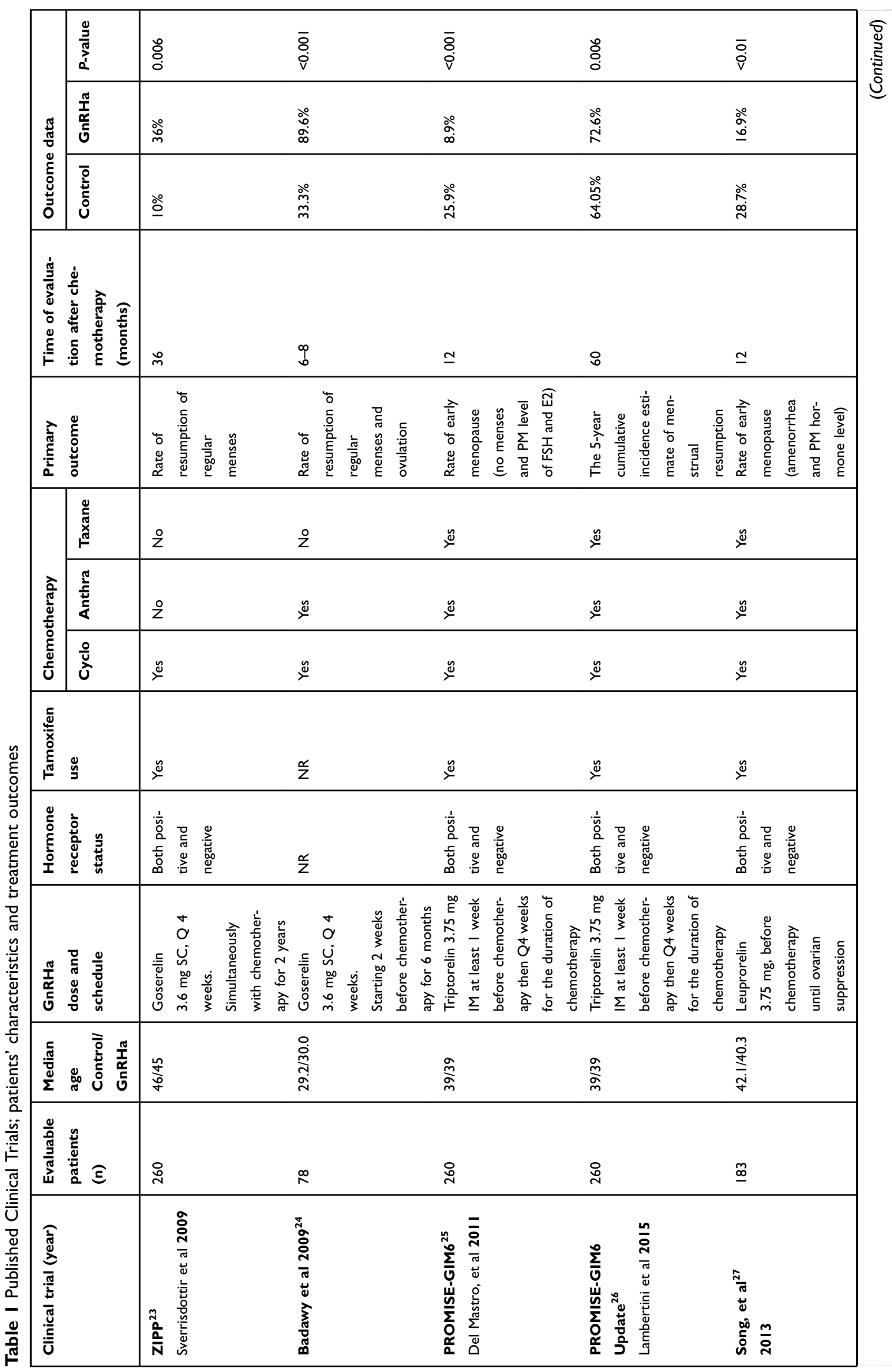







$P<0.001)$. FSH and LH levels were also significantly lower in the GnRH agonist group. ${ }^{24}$

The PROMISE-GIM6 (Prevention of Menopause Induced by Chemotherapy: A Study in Early Breast Cancer PatientsGruppo Italiano Mammella-6) study was one of the largest studies in this field. Two hundred eighty-one (260 evaluable) premenopausal women with stage I-III breast cancer who were candidates for adjuvant or neoadjuvant chemotherapy were enrolled. Patients were randomized to receive chemotherapy alone or combined with triptorelin administered at least one week before the start of chemotherapy and then every four weeks for the duration of chemotherapy. Twelve months after the last cycle of chemotherapy, the incidence of early menopause (defined as no resumption of menstrual activity and postmenopausal levels of FSH and estradiol 1 year after the last cycle of chemotherapy) was $25.9 \%$ in the chemotherapy-alone group and $8.9 \%$ in the chemotherapy plus triptorelin group, an absolute difference of $17 \%(95 \% \mathrm{CI}$, $-26 \%$ to $-7.9 \% ; P<0.001)$. The odds ratio for treatmentrelated early menopause was 0.28 (95\% CI, $0.14-0.59$; $P<0.001) .{ }^{25}$ The study was updated a few years later; the 5 -year cumulative incidence of menstrual resumption was $72.6 \%$ (95\% CI, 65.7-80.3\%) in the GnRH agonist group and $64.0 \%$ (95\% CI, 56.2-72.8\%) in the control group (HR, 1.28 [95\% CI, 0.98-1.68]; $P=0.07$ ); age-adjusted HR, 1.48 (95\% CI, 1.12-1.95, $P=0.006$ ). There was no statistical difference in pregnancy rates between the groups. The authors also reported on 5-year DFS; $80.5 \%$ (95\% CI, 73.1-86.1\%) in the GnRH agonist group and $83.7 \%$ (95\% CI, 76.1-89.1\%) in the control group (GnRH agonist vs control: HR, 1.17 [95\% CI, 0.72-1.92]; $P=0.52){ }^{26}$

A Chinese study included a total of 183 evaluable patients who were randomized to receive cyclophosphamide and doxorubicin-based chemotherapy alone or chemotherapy plus leuprolide. The primary endpoint was resumption of menses or premenopausal levels of both FSH and E2 within 12 months after the end of chemotherapy. At the end of follow-up, $41.5 \%$ in the chemotherapy group and $59.6 \%$ in the chemotherapy plus leuprolide group resumed menses $(P<0.05)$. Additionally, $7.4 \%$ in the chemotherapy group and $15.7 \%$ in the chemotherapy plus leuprolide group had restored premenopausal levels of FSH and E2. The median time to resume menses was 9.2 months for patients in the chemotherapy plus leuprolide group and was not reached in the chemotherapy-only group. Additionally, this study demonstrated that age had no significant effect on the occurrence of premature menopause. $^{27}$
The OPTION trial, one of the most recently published prospective, randomized trials, enrolled 227 patients (202 evaluable) with early-stage breast cancer regardless of their hormone receptor status, and they were randomized to receive chemotherapy with or without goserelin, which was started at least one week before chemotherapy and then every four weeks. The primary endpoint was premature ovarian insufficiency (POI), defined as amenorrhea between 12 and 24 months after randomization with elevated FSH. Goserelin reduced the prevalence of amenorrhea to $22 \%$ compared to $38 \%$ in the control group $(P=0.015)$ and the prevalence of POI to $18.5 \%$ compared to $34.8 \%$ in the control group $(P=0.048)$. However, the effect of goserelin was not statistically significant in women $>40$ years (amenorrhea: $42.9 \%$ vs $54.2 \%, P=0.376$ ). ${ }^{28}$

Despite the many previously discussed "positive" studies, several others failed to demonstrate any advantage for the GnRH agonists. In one negative study, women aged 44 years or younger (median: 39 years) were randomly assigned to receive their adjuvant or neoadjuvant chemotherapy with or without triptorelin given at least one week before chemotherapy and then every four weeks throughout the chemotherapy duration. The chemotherapy was anthracycline-based with or without four additional cycles of taxanes. Patients were stratified by chemotherapy regimen used, age $(<35,35-39,>39$ years $)$ and estrogen receptor status. Primary objectives included the resumption of menses at least 2 years after the last cycle of chemotherapy. Follicle-stimulating hormone and inhibin $\mathrm{A}$ and $\mathrm{B}$ levels were also monitored during the follow-up period. Although the study was planned to recruit 124 patients with a planned 5-year follow-up, the trial was stopped prematurely for futility after 49 patients were enrolled (47 evaluable). Menstruation resumed in 19 $(90 \%)$ of 21 patients in the control group and in 23 $(88 \%)$ of 26 in the triptorelin group $(P=0.36)$. Median time for resumption of menses was 5.8 months after completion of chemotherapy in the triptorelin group versus 5.0 months in the control arm $(P=0.58)$. Both FSH and inhibin $\mathrm{B}$ levels correlated with menstrual status. ${ }^{29}$

The Egyptian study by Elgindy et al was another negative trial but used a different approach by giving both GnRH agonists and antagonists at the same time, and as such, its results might not reflect what had been done in all other clinical trials. ${ }^{30}$

Two other studies restricted their enrollment to hormone receptor-negative patients. In the POEMS/S0230 study, 257 premenopausal women with early-stage, 
hormone-receptor-negative breast cancer were randomized to receive standard chemotherapy with or without the GnRH agonist goserelin. The rate of ovarian failure at 2 years was the primary study endpoint. Ovarian failure was defined as the absence of menses in the preceding 6 months and levels of FSH in the postmenopausal range. Pregnancy outcomes and DFS and OS were secondary endpoints. Only 135 patients had complete primary endpoint data. The ovarian failure rate was $8 \%$ in the goserelin group and $22 \%$ in the chemotherapy-alone group (odds ratio, 0.30; 95\% CI, 0.09-0.97; $P=0.04$ ). Among the 218 patients who could be evaluated, pregnancy occurred in $21 \%$ in the goserelin group compared to $11 \%$ in the chemotherapy-alone group, $P=0.03$. Additionally, both DFS $(P=0.04)$ and $\operatorname{OS}(P=0.05)$ were better in the goserelin group. ${ }^{31}$

The other study, GBG-37 ZORO, was a randomized, open-label, controlled multicenter study in which 60 patients younger than 46 years, all with hormonereceptor negative breast cancer, were randomized to receive chemotherapy with or without goserelin. The chemotherapy was anthracycline/cyclophosphamide (with or without taxanes) and was given in a neoadjuvant setting. Patients received goserelin at least 2 weeks before the first cycle of chemotherapy and then every 4 weeks until the end of the last cycle. The primary endpoint was the rate of reappearance of normal ovarian function, defined as two consecutive menstrual cycles within 21-35 days at 6 months after chemotherapy. At the time of evaluation, normal ovarian function was observed in 70.0\% [95\% CI, 53.6-86.4\%] in the goserelin group compared to $56.7 \%$ [95\% CI, 39-74.4\%] in the control group [difference, $13.3 \%$; 95\% CI, $-10.85-37.45 ; P=0.284]$. $^{32}$

\section{Meta-analyses}

Given the conflicting results of the published studies, the relatively small number of patients included and the many variables that may affect ovarian function recovery following chemotherapy, several meta-analyses were conducted addressing this question.

Del Mastro et al conducted a meta-analysis that included nine studies with 765 evaluable cancer patients that included lymphoma and ovarian cancer in addition to breast cancer. The pooled OR estimate showed a highly significant reduction in the risk of POF (OR 0.43; 95\% CI: $0.22-0.84 ; P=0.013$ ) in patients receiving $\mathrm{GnRH}$ agonists. Subgroup analysis showed that the protective effect of GnRH agonists against POF was present for breast cancer but unclear in ovarian cancer and lymphoma patients. The protective effect was also similar in subgroups of patients defined by age and timing of POF assessment. ${ }^{33}$

The same group led by Lambertini performed another systematic review and meta-analysis on the basis of abstracted data from 12 eligible randomized clinical trials that included a total of 1,231 patients, all with early-stage breast cancer. The use of GnRH agonists was associated with a significant reduction in the risk of POF (OR 0.36, 95\% CI $0.23-0.57 ; P<0.001)$. In the five studies that reported pregnancies, more patients treated with $\mathrm{GnRH}$ agonists achieved pregnancy (33 versus 19 women; OR $1.83,95 \%$ CI $1.02-3.28, P=0.041)$. No difference was observed in DFS in the three studies that reported this outcome (HR 1.00, 95\% CI 0.49-2.04, $P=0.939$ ). ${ }^{34}$

In another meta-analysis that included seven studies totaling 856 evaluable patients, the use of GnRH agonists was associated with a higher rate of recovery of regular menses at 6 months (OR, 2.41; 95\% CI, 1.40-4.15; $P=0.002)$ and at 12 months (OR, 1.85; 95\% CI, $1.33-2.59 ; \quad P<0.001)$ following the last chemotherapy cycle. Though pregnancy was not uniformly reported and the rate of pregnancy was not the primary outcome in any of the trials, the use of GnRH agonists was also associated with a higher number of pregnancies (OR, 1.85; 95\% CI, $1.02-3.36 ; P=0.04) .^{35}$

Despite the many positive meta-analyses discussed above, Elgindy et al reported a negative one. In their metaanalysis, 10 eligible trials with 907 women with breast, lymphoma and ovarian cancer were included. GnRH agonists failed to show significant increases in ovarian function resumption in the GnRH agonists arm (68.4\%) and in the chemotherapy alone arm (59.9\%) [OR, 1.12, 95\% CI, 0.99-1.27]. No protective effect existed after subgroup analysis [age $(P=0.14)$, GnRH agonists type $(P=0.44)$ or type of malignancy $(P=0.310)]$. In breast cancer patients, in particular, resumed ovarian function was reported in a total of $207(57.5 \%)$ of 360 in the chemotherapy-alone arm compared to $251(65 \%)$ in the GnRH agonist arm $(P=0.25){ }^{36}$

More recently Lambertini et al used individual patientlevel data to include a total of 873 patients from five trials in a meta-analysis. The five trials included were PROMISE-GIM $6,{ }^{25}$ OPTION,$^{28}$ the Moffitt-led trial, ${ }^{29}$ POEMS $^{31}$ and ZORO. $^{32}$ Outcome data were available for $722(82.7 \%)$ of 873 patients. In the $\mathrm{GnRH}$ agonist-treated group $(n=363), 51$ (14.1\%) developed POI compared with 111 (30.9\%) in the control group $(n=359)$ [adjusted OR, 0.38 ; 95\% CI, 0.26-0.57; 
$P=0.001]$. The effect of GnRH agonists on reducing the risk of developing chemotherapy-induced POI was homogeneous among the different patient subgroups. On multivariate analysis, only younger age at diagnosis (adjusted OR, 0.35; 95\% CI, $0.24-0.52 ; P=0.001)$ and treatment with $\mathrm{GnRH}$ agonists (adjusted OR, 0.38; 95\% CI, 0.26-0.57; $P=0.001$ ) were significantly associated with a reduced risk of developing chemotherapy-induced POI. ${ }^{37}$

Another meta-analysis that reviewed 15 published studies looked into several patient- and treatment-related factors associated with ovarian function recovery after chemotherapy. Five different factors were analyzed: coadministration of $\mathrm{GnRH}$ agonists, addition of taxanes to anthracycline-based chemotherapy and addition of endocrine therapy to chemotherapy (treatment-related factors). Additionally, they looked at age and baseline levels of anti-Müllerian hormone (patient-related factors). Recovery of menses was the most widely used marker. Younger age ( $\leq 40$ years) and exposure to GnRH agonists were positively associated with menses recovery (OR 6.07 and 2.03, respectively), while exposure to taxanes adversely affected recovery (OR 0.49). ${ }^{38}$

\section{Pregnancy as an endpoint}

As discussed above, pregnancy rate was not a primary endpoint in any of the discussed clinical trials. However, it was among many other secondary endpoints. Given the small number of patients included and the short follow-up, it is extremely difficult to make solid conclusions.

In one retrospective cohort study, 286 patients who received GnRH agonists with chemotherapy were compared to 188 patients who were treated with chemotherapy alone. Ovarian function could be determined in 217 patients. Overall, 127 (87\%) of 146 patients in the GnRH agonist group retained cyclic ovarian function compared to $35(49 \%)$ of 71 patients in the control group $(P=0.0001$, OR 6.87, 95\% CI 3.4-13.4). Pregnancy was reported in 84 $(69.3 \%)$ of 122 patients in the GnRH agonist group compared to $28(42.4 \%)$ of 66 patients in the control group $(P=0.006)$. Spontaneous pregnancies occurred significantly more frequently in the GnRH agonist group; $65.6 \%$ versus $37.9 \%\left(P=0.0004\right.$, OR 3.12, 95\% CI 1.7-5.8). ${ }^{39}$

\section{Safety issues}

GnRH agonists result in a hypoestrogenic state that can be achieved in almost three-quarters of women treated with leuprolide within the first four weeks of therapy and almost all by eight weeks. ${ }^{40}$ Obviously, patients may experience vasomotor symptoms, hot flashes, sleep disturbances, vaginal dryness and urogenital atrophy.

The potential for bone loss could also be a concern, as accelerated bone loss following both chemotherapy and hormonal therapy is not uncommon, especially in young premenopausal women.

\section{International guidelines}

The issue of fertility preservation, including the use of GnRH agonists, among young women treated for cancer has been addressed by several regional and international guidelines.

The latest ASCO updated guidelines recommend that GnRH agonists only be offered in the setting of young women with breast cancer when proven fertility preservation methods are not feasible. ${ }^{13}$ Like the ASCO, the European Society of Medical Oncology (ESMO) guidelines do not recommend GnRH agonists as a method of fertility preservation. ${ }^{41}$ Additionally, the National Comprehensive Cancer Network (NCCN), in both "adolescent and young adult oncology"42 and "breast cancer", 43 does not recommend GnRH agonists and expressed concerns about the inconsistency of the published trials and meta-analyses and the limited data on the long-term impact of GnRH agonists on preservation of ovarian function. However, the Spanish guideline, ${ }^{44}$ SEOM, stated that GnRH agonists could be an option to discuss with patients with early-stage hormone-receptornegative breast cancer if embryo or oocyte cryopreservation is not feasible, while the St. Gallen International Consensus strongly supported the use of OFS during chemotherapy for hormone-receptor-negative disease to preserve ovarian function and fertility. ${ }^{45}$ Additionally, both the Italian Association of Medical Oncology ${ }^{46}$ and the Second International Consensus Guidelines for Breast Cancer in Young Women (BCY2) ${ }^{47}$ had positive recommendations as well.

\section{Conclusion and future directions}

Given the better awareness, early detection and recent advances in breast cancer treatment, more younger women will survive their disease, highlighting the importance of structured patient education and survivorship programs that deal with late toxicities associated with cancer therapy. Formal oncofertility clinics with better communication between the treating oncologist and fertility experts can improve patient access to fertilitypreservation options and may result in an improved 
physical and psychological quality of life. ${ }^{12,48,49}$ Standardized referral to such programs and clinics could be a key performance indicator. ${ }^{14}$

Given the established rules of fertilized and unfertilized ova preservation and the recent advances in ovarian tissue cryopreservation, GnRH agonists should not be used in place of such proven methods. Data on GnRH agonists are conflicting, and surrogate markers used in many of the discussed trials do not necessarily reflect successful pregnancies. Live birth rate data, generated from long-term follow-up, are the most appropriate marker of fertility. Few studies have included enough patients or had a sufficient length of follow-up to make definitive conclusions. Reliance on surrogate markers, such as resumption of menses or hormonal levels, might not be the best endpoint. As such, GnRH agonists should only be used in situations where proven methods cannot be used. However, such drugs can, in the long term, minimize symptoms and complications related to POI.

\section{Acknowledgments}

The author would like to thank Mrs. Alice Haddadin, Ms. Haifa Al-Ahmad and Mrs. Laila Alqtua for their great support in preparing this manuscript.

\section{Disclosure}

The author reports no conflicts of interest in this work.

\section{References}

1. Ghoncheh M, Pournamdar Z, Salehiniya H. Incidence and mortality and epidemiology of breast cancer in the world. Asian Pac J Cancer Prev. 2016;17(S3):43-46.

2. Cancer today; 2018. Available from: http://globocan.iarc.fr/old/ FactSheets/cancers/breast-new.asp. Accessed November 1, 2018.

3. Abdel-Razeq H, Attiga F, Mansour A. Cancer care in Jordan. Hematol Oncol Stem Cell Ther. 2015;8(2):64-70. doi:10.1016/j. hemonc.2015.02.001

4. Tichy JR, Lim E, Anders CK. Breast cancer in adolescents and young adults: a review with a focus on biology. J Natl Compr Canc Netw. 2013;11(9):1060-1069.

5. Gewefel H, Salhia B. Breast cancer in adolescent and young adult women. Clin Breast Cancer. 2014;14(6):390-395. doi:10.1016/j. clbc.2014.06.002

6. Moore HCF. Fertility and the impact of systemic therapy on hormonal status following treatment for breast cancer. Curr Oncol Rep. 2000;2:587-593. doi:10.1007/s11912-000-0114-9

7. Bines J, Oleske DM, Cobleigh MA. Ovarian function in premenopausal women treated with adjuvant chemotherapy for breast cancer. J Clin Oncol. 1996;14(5):1718-1729. doi:10.1200/JCO.1996.14.5.1718

8. Anderson C, Engel SM, Anders CK, Nichols HB. Live birth outcomes after adolescent and young adult breast cancer. Int J Cancer. 2018;142 (10):1994-2002. doi:10.1002/ijc.31227
9. Goodwin PJ, Ennis M, Pritchard KI, Trudeau M, Hood N. Risk of menopause during the first year after breast cancer diagnosis. $J$ Clin Oncol. 1999;17(8):2365-2370. doi:10.1200/JCO.1999.17.8.2365

10. Fornier MN, Modi S, Panageas KS, Norton L, Hudis C. Incidence of chemotherapy-induced, long-term amenorrhea in patients with breast carcinoma age 40 years and younger after adjuvant anthracycline and taxane. Cancer. 2005;104(8):1575-1579. doi:10.1002/cncr.21385

11. Takai Y. Recent advances in oncofertility care worldwide and in Japan. Reprod Med Biol. 2018;17(4):356-368. doi:10.1002/ $\mathrm{rmb} 2.12214$

12. Kelvin JF, Thom B, Benedict C, et al. Cancer and fertility program improves patient satisfaction with information received. J Clin Oncol. 2016;34(15):1780-1786. doi:10.1200/JCO.2015.64.5168

13. Oktay K, Harvey BE, Partridge A, et al. Fertility preservation in patients with cancer: ASCO clinical practice guideline update. J Clin Oncol. 2018;36(19):1994-2001. doi:10.1200/JCO.2018.78.1914

14. McCray DK, Simpson AB, Flyckt R, et al. Fertility in women of reproductive age after breast cancer treatment: practice patterns and outcomes. Ann Surg Oncol. 2016;23(10):3175-3181. doi:10.1245/ s10434-016-5308-y

15. Benedict C, Thom B, N Friedman D, et al. Young adult female cancer survivors' unmet information needs and reproductive concerns contribute to decisional conflict regarding post treatment fertility preservation. Cancer. 2016;122:2101-2109. doi:10.1002/cncr.29917

16. Schally AV, Coy DH, Arimura A. LH-RH agonists and antagonists. Int $J$ Gynaecol Obstet. 1980;18(5):318. doi:10.1002/j.18793479.1980.tb00507.x

17. Conn PM, Crowley WF Jr. Gonadotropin-releasing hormone and its analogues. $N$ Engl J Med. 1991;324(2):93. doi:10.1056/ NEJM199101103240205

18. Rivkees SA, Crawford JD. The relationship of gonadal activity and chemotherapy-induced gonadal damage. JAMA. 1988;259 (14):2123-2125.

19. Ataya K, Rao LV, Lawrence E, et al. Luteinizing hormone-releasing hormone agonist inhibits cyclophosphamide-induced ovarian follicular depletion in rhesus monkeys. Biol Reprod. 1995;52:365-372. doi:10.1095/biolreprod52.2.365

20. Lobo RA. Potential options for preservation of fertility in women. $N$ Engl J Med. 2005;353:64-73. doi:10.1056/NEJMra043475

21. Blumenfeld Z. How to preserve fertility in young women exposed to chemotherapy? The role of GnRH agonist cotreatment in addition to cryopreservation of embrya, oocytes, or ovaries. Oncologist. 2007;12 (9):1044-1054. doi:10.1634/theoncologist.12-9-1044

22. Kitajima Y, Endo T, Nagasawa K, et al. Hyperstimulation and a gonadotropin-releasing hormone agonist modulate ovarian vascular permeability by altering expression of the tight junction protein claudin-5. Endocrinology. 2006;147:694-699. doi:10.1210/en.20050700

23. Sverrisdottir A, Nystedt M, Johansson H, Fornander T. Adjuvant goserelin and ovarian preservation in chemotherapy treated patients with early breast cancer: results from a randomized trial. Breast Cancer Res Treat. 2009;117:561-567. doi:10.1007/s10549-009-0313-5

24. Badawy A, Elnashar A, El-Ashry M, et al. Gonadotropin-releasing hormone agonists for prevention of chemotherapy-induced ovarian damage: prospective randomized study. Fertil Steril. 2009;91:694-697. doi:10.1016/j.fertnstert.2007.12.044

25. Del Mastro L, Boni L, Michelotti A, et al. Effect of the gonadotropin releasing hormone analogue triptorelin on the occurrence of chemotherapy-induced early menopause in premenopausal women with breast cancer: a randomized trial. JAMA. 2011;306:269-276. doi:10.1001/jama.2011.991

26. Lambertini M, Boni L, Michelotti A, et al.; for the GIM Study Group. Ovarian suppression with Triptorelin during adjuvant breast cancer chemotherapy and long-term ovarian function, pregnancies, and disease-free survival: a Randomized Clinical Trial. JAMA. 2015;314:2632-2640. doi:10.1001/jama.2015.17291 
27. Song G, Gao H, Yuan Z. Effect of leuprolide acetate on ovarian function after cyclophosphamide-doxorubicin-based chemotherapy in premenopausal patients with breast cancer: results from a phase II randomized trial. Med Oncol. 2013;30:667. doi:10.1007/s12032-013-0667-8

28. Leonard RCF, Adamson DJA, Bertelli G, et al; Anglo Celtic Collaborative Oncology Group and National Cancer Research Institute Trialists. GnRH agonist for protection against ovarian toxicity during chemotherapy for early breast cancer: the Anglo Celtic Group OPTION trial. Ann Oncol. 2017;28:1811-1816.

29. Munster PN, Moore AP, Ismail-Khan R, et al. Randomized trial using gonadotropin-releasing hormone agonist triptorelin for the preservation of ovarian function during (neo)adjuvant chemotherapy for breast cancer. J Clin Oncol. 2012;30:533-538. doi:10.1200/JCO.2011.34.6890

30. Elgindy EA, El-Haieg DO, Khorshid OM, et al. Gonadatrophin suppression to prevent chemotherapy-induced ovarian damage: a randomized controlled trial. Obstet Gynecol. 2013;121:78-86. doi:10.1097/AOG.0b013e31827374e2

31. Moore HC, Unger JM, Phillips KA, et al.; POEMS/S0230 Investigators. Goserelin for ovarian protection on during breast-cancer adjuvant chemotherapy. $N$ Engl J Med. 2015;372:923-932. doi:10.1056/ NEJMoa1413204

32. Gerber B, von Minckwitz G, Stehle H, et al.; German Breast Group Investigators. Effect of luteinizing hormone-releasing hormone agonist on ovarian function on after modern adjuvant breast cancer chemotherapy: the GBG 37 ZORO study. $J$ Clin Oncol. 2011;29:2334-2341. doi:10.1200/JCO.2010.32.5704

33. Del Mastro L, Ceppi M, Poggio F, et al. Gonadotropin-releasing hormone analogues for the prevention of chemotherapy-induced premature ovarian failure in cancer women: systematic review and meta-analysis of randomized trials. Cancer Treat Rev. 2014;40:675-683. doi:10.1016/j.ctrv.2013.12.001

34. Lambertini M, Ceppi M, Poggio F, et al. Ovarian suppression using luteinizing hormone releasing hormone agonists during chemotherapy to preserve ovarian function and fertility of breast cancer patients: A meta-analysis of randomized studies. Ann Oncol. 2015;26:2408-2419. doi:10.1093/annonc/mdv374

35. Munhoz RR, Pereira AA, Sasse AD, et al. Gonadotropin-releasing hormone agonists for ovarian function preservation in premenopausal women undergoing chemotherapy for early-stage breast cancer: a systematic review and meta-analysis. JAMA Oncol. 2016;2:65-73. doi:10.1001/jamaoncol.2015.3251

36. Elgindy E, Sibai H, Abdelghani A, Mostafa M. Protecting ovaries during chemotherapy through gonad suppression: a systematic review and meta-analysis. Obstet Gynecol. 2015;126:187-195. doi:10.1097/ AOG.0000000000000905

37. Lambertini M, Moore HCF, Leonard RCF, et al. Gonadotropinreleasing hormone agonists during chemotherapy for preservation of ovarian function and fertility in premenopausal patients with early breast cancer: a systematic review and meta-analysis of individual patient-level data. J Clin Oncol. 2018;36:1981-1990. doi:10.1200/ JCO.2018.78.0858
38. Silva C, Caramelo O, Almeida-Santos T, Ribeiro Rama AC. Factors associated with ovarian function recovery after chemotherapy for breast cancer: a systematic review and meta-analysis. Hum Reprod. 2016;31(12):2737-2749. doi:10.1093/humrep/dew224

39. Blumenfeld Z, Zur H, Dann EJ. Gonadotropin-releasing hormone agonist cotreatment during chemotherapy may increase pregnancy rate in survivors. Oncologist. 2015;20(11):1283-1289. doi:10.1634/ theoncologist.2015-0223

40. Hornstein MD, Surrey ES, Weisberg GW, Casino LA; Lupron AddBack Study Group. Leuprolide acetate depot and hormonal add-back in endometriosis: a 12-month study. Obstet Gynecol. 1998;91(1):16. doi:10.1016/S0029-7844(97)00620-0

41. Paluch-Shimon S, Pagani O, Partridge AH, et al. ESO-ESMO 3rd international consensus guidelines for breast cancer in young women (BCY3). Breast. 2017;35:203-217. doi:10.1016/j. breast.2017.07.017

42. National Comprehensive Cancer Network. National Comprehensive Cancer Network Guidelines Adolescent and Young Adult (AYA) Oncology; 2018. Available from: https://www.nccn.org/profes sionals/physician_gls/pdf/aya.pdf. Accessed November 1, 2018.

43. National Comprehensive Cancer Network. National Comprehensive Cancer Network Guidelines - Breast Cancer; 2018. Available from: https://www.nccn.org/professionals/physician_gls/pdf/breast.pdf. Accessed November 1, 2018.

44. Muñoz M, Santaballa A, Segui' MA, et al. SEOM Clinical Guideline of fertility preservation and reproduction in cancer patients. Clin Transl Oncol. 2016;18:1229-1236. doi:10.1007/ s12094-016-1587-9

45. Coates AS, Winer EP, Goldhirsch A, et al. Tailoring therapiesimproving the management of early breast cancer: St Gallen International Expert Consensus on the primary therapy of early breast cancer 2015. Ann Oncol. 2015;26:1533-1546. doi:10.1093/annonc/ mdv221

46. Lambertini M, Cinquini M, Moschetti I, et al. Temporary ovarian suppression during chemotherapy to preserve ovarian function and fertility in breast cancer patients: a GRADE approach for evidence evaluation and recommendations by the Italian Association of Medical Oncology. Eur J Cancer. 2017;71:25-33. doi:10.1016/j. ejca.2016.10.034

47. Paluch-Shimon S, Pagani O, Partridge AH, et al. Second international consensus guidelines for breast cancer in young women (BCY2). Breast. 2016;26:87-99. doi:10.1016/j.breast.2015.12.010

48. Vu JV, Llarena NC, Estevez SL, Moravek MB, Jeruss JS. Oncofertility program implementation increases access to fertility preservation options and assisted reproductive procedures for breast cancer patients. J Surg Oncol. 2017;115:116-121. doi:10.1002/ jso. 24418

49. Deshpande NA, Braun IM, Meyer FL. Impact of fertility preservation counseling and treatment on psychological outcomes among women with cancer: a systematic review. Cancer. 2015;121:3938-3947. doi:10.1002/cncr.29637

\section{Publish your work in this journal}

Cancer Management and Research is an international, peer-reviewed open access journal focusing on cancer research and the optimal use of preventative and integrated treatment interventions to achieve improved outcomes, enhanced survival and quality of life for the cancer patient.
The manuscript management system is completely online and includes a very quick and fair peer-review system, which is all easy to use. Visit http://www.dovepress.com/testimonials.php to read real quotes from published authors. 